\title{
Distributed Transmit Antenna Selection (DTAS) under Performance or Energy Consumption Constraints
}

\author{
Diomidis S. Michalopoulos, Student Member, IEEE, George K. Karagiannidis, Senior Member, IEEE, \\ Theodoros A. Tsiftsis, Member, IEEE, and Ranjan K. Mallik, Senior Member, IEEE
}

\begin{abstract}
Motivated by the transmit antenna selection (TAS) concept, used in Multiple-Input-Multiple-Output systems, we argue for distributed transmit antenna selection (DTAS), which corresponds to a method of selecting a subset of available relays in cooperative diversity systems. Assuming amplify and forward relays, the proposed selection method represents a low-complexity tool for determining the optimum relaying set. Two optimization problems are studied: the error probability minimization subject to total energy consumption constraints, and the dual one, the total energy consumption minimization under error performance constraints. Numerical examples verify the advantage of the proposed method in adapting the number of relaying terminals to the desired performance-consumption tradeoff.
\end{abstract} tion.

Index Terms-Cooperative diversity, transmit antenna selec-

\section{INTRODUCTION}

$\mathbf{T}$ RANSMITTING from only a subset of the set of available transmitting antennas is a concept that has gained increasing interest, since it attains a reduction in the transmitting power while still achieving the beneficial effects of spatial diversity. Such systems are generally referred in the literature as transmit antenna selection (TAS) [1]- [3], the basic characteristic of which is that the multiple transmitting antennas are co-located, i.e., they are carried by the same terminal. However, research on the recently appeared topic of cooperative diversity demonstrate [4]- [8] that spatial diversity can also be achieved with a single transmit and receive antenna, by employing spatially-separated relaying terminals which actually form a virtual antenna array. Therefore, TAS systems can also be studied and thereby designed from a distributed perspective, forming distributed transmit antenna selection (DTAS) systems, where only a subset of the available relays needs to be selected according to certain criteria.

Manuscript received November 16, 2006; revised March 28, 2007; accepted June 4,2007 . The editor coordinating the review of this paper and approving it for publication was T. M. Duman. This work was conducted within the framework of the Reinforcement Program of Human Research Manpower (PENED'03), partially funded by the E.U.-European Social Fund (75\%) and the Greek Ministry of Development (25\%). This paper was presented in part at IEEE Global Communications Conference (GLOBECOM'06), San Francisco, California, USA, November-December 2006.

D. S. Michalopoulos, G. K. Karagiannidis, and T. A. Tsiftsis are with the Department of Electrical and Computer Engineering, Aristotle University of Thessaloniki, GR-54124 Thessaloniki, Greece (e-mail: \{dmixalo, geokarag, thtsif\}@auth.gr).

R. K. Mallik is with the Department of Electrical Engineering, Indian Institute of Technology - Delhi, Haus Khas, New Delhi 110016, India (email: rkmallik@ee.iitd.ernet.in).

Digital Object Identifier 10.1109/TWC.2008.060950.
The concept of single relay selection among a set of available ones (which actually represents a special case of the general DTAS problem) has recently attracted the attention of many researchers working in this field. Several selection criteria were proposed, including selection of the relay that leads to the minimum asymptotic symbol error probability (SEP) [8] and selection of the relay that corresponds to the best average channel conditions [9] or the instantaneous ones [9][11]. In [10] it was shown that "opportunistic relaying", which is simpler to implement than distributed space-time coding (DSTC), results in the same diversity-multiplexing tradeoff as that of DSTC. Later on, the authors of [11] showed that this method outperforms DSTC in terms of outage probability. The asymptotic SEP of single-selection amplify and forward (AF) relaying schemes has been studied in [12], where it was shown that single DTAS outperforms the scheme where all the available relays participate in the relaying process, assuming the same total transmitting power in both schemes. Furthermore, a specific type of DTAS for decode and forward relaying was proposed in [13], where the authors developed a distributed beamforming technique that significantly improves the performance while taking into account the energy consumption and complexity involved.

In this letter, we propose a novel DTAS strategy, according to which only a subset of the set of the available AF relaying terminals is activated, in order to achieve a well-balanced tradeoff between error performance and total consumed energy. The selection is done according to average channel conditions, and represents a low-complexity tool for determining the optimum relaying set. In particular, two variations are introduced: The end-to-end error performance optimization under total energy consumption constraints, and the dual one, the minimization of the total consumed energy provided that the end-to-end error probability does not exceed a predefined threshold. This is attained by utilizing the general concept of optimizing the selection among the elements of a given set under specific constraints, which was first introduced in combinatorial optimization theory: Given an item set, with a unique pair of profit and weight values attributed to each item, the subset that maximizes the profit summation provided that the weight summation does not exceed a maximum value needs to be distinguished. These optimization problems are known as knapsack problems [14].

\section{SYSTEM MODEL}

We consider a source node $S$ communicating with a destination node $D$ with the aid of $L$ other relaying nodes, 
denoted by $R_{j}, \quad j \in\{1, \ldots, L\}$, each one employing a single transmit/receive antenna. The relays operate in the non-regenerative mode, i.e., they amplify and retransmit the received signal without demodulating it. Also, in order to satisfy the half-duplex constraint, the relays are assumed to transmit and receive in different time slots. Each transmission period is thus divided into two sub-periods: In the former, the source communicates with the relays and the destination terminal, while in the latter, only the relays communicate with the destination, each one using a separate orthogonal channel. This relaying model was also considered in [7]- [8], [15].

In the proposed model, the destination is assumed to have full knowledge of all the average $R_{j}-D$ and $S-R_{j}$, $j \in\{1, \ldots, L\}$, channel conditions. Hence, the selection is performed at $D$ during the initialization stage (before the communication begins), and the selected relays remain activated as long as the fading conditions do not significantly change, in an average sense. Notice that no continuous channel estimation is needed; depending on the propagation environment, the average fading conditions can be estimated using a long training sequence, and continuously improved during the communication period (see also [16]). We should state, however, that the destination is assumed to employ a maximal ratio combiner (MRC); this implies that the selected relays need to estimate the channel at their input and then, together with the forwarded data, pass the $S-R_{j}$ channel state information (CSI) to $D$, so as, together with the $R_{j}-D$ CSI, $D$ can appropriately combine the received signals into the MRC.

Let $P_{S}$ represent the source's transmitting power ${ }^{1}$. The gain $G_{j}$ of $R_{j}$ aims at limiting the relay's output power [4] i.e.,

$$
G_{j}^{2}(t)=\frac{P_{j, \text { out }}}{P_{S} a_{S j}^{2}(t)+N_{0}},
$$

where $P_{j, \text { out }}$ is the relay's transmission power, $a_{S j}(t)$ is the fading amplitude of the $S-R_{j}$ channel and $N_{0}$ stands for the additive white Gaussian noise (AWGN) power, which is assumed identical in each link. The instantaneous signal-tonoise-ratio (SNR), $\gamma_{j}$, of the $b_{j}$ branch (where a branch here is defined as an end-to-end communication path $\left.S-R_{j}-D\right)$ is given by [4]

$$
\gamma_{j}=\frac{\gamma_{S j} \gamma_{D j}}{\gamma_{S j}+\gamma_{D j}+1}
$$

where $\gamma_{S j}, \gamma_{D j}$ are the instantaneous SNRs of the $S-R_{j}$ and $R_{j}$-D link, respectively. Assuming that $l$ nodes are operating during a given transmission period, the overall instantaneous SNR at the output of the MRC at $D$ during this period can be written as

$$
\gamma_{e n d}=\gamma_{0}+\sum_{j=1}^{l} \frac{\gamma_{S j} \gamma_{D j}}{\gamma_{S j}+\gamma_{D j}+1}=\sum_{j=0}^{l} \gamma_{j},
$$

where $\gamma_{0}$ is the instantaneous SNR of the direct $S-D$ channel. The branch corresponding to the $S-D$ channel is denoted by $b_{0}$.

\footnotetext{
${ }^{1}$ Without loss of generality, we assume constant $P_{S}$, although this model is also applied when non-constant envelope modulations are used; in such case, $P_{S}$ represents the average transmitting power over the variable symbol amplitudes.
}

\section{ThE KNAPSACK PROBlem AND ITS APPLICATION ON DTAS SYSTEMS}

The well-known zero-one knapsack problem is defined as follows [14]:

Given an item set $\mathcal{N}$, consisting of $L$ items with profits $p_{j}>0$ and weights $w_{j}>0, j=1, \ldots, L$, and given the capacity value $C_{\max }$, select the subset of $\mathcal{N}$ such that the total profit of the selected items is maximized while the total weight does not exceed $C_{\max }$. In other words,

$$
\begin{gathered}
\text { maximize } \sum_{j=1}^{L} p_{j} x_{j} \\
\text { subject to } \sum_{j=1}^{L} w_{j} x_{j} \leq C_{\max }, \\
x_{j} \in\{0,1\}, \quad j=1, \ldots, L .
\end{gathered}
$$

A variation of this problem is to minimize (instead of maximize) the profit summation, under the constraint that the total weight is greater than or equal to a given value $C_{\min }$, i.e.,

$$
\begin{gathered}
\text { minimize } \sum_{j=1}^{L} p_{j} y_{j} \\
\text { subject to } w_{0}+\sum_{j=1}^{L} w_{j} y_{j} \geq C_{\min }, \\
y_{j} \in\{0,1\}, \quad j=1, \ldots, L,
\end{gathered}
$$

Throughout this letter, we refer to the problems having the form of (4) as traditional knapsack problems, while to the ones with the form of (5) as minimization knapsack problems.

1) Efficient Knapsack Algorithms: One efficient suboptimal knapsack algorithm is the well-known Greedy one, which operates as follows [14]:

Algorithm 1 (Traditional Knapsack problem): "For every item $j \in \mathcal{N}$, denote with $e_{j}$ the profit to weight ratio, which is also called the efficiency of this item, i.e.,

$$
e_{j}:=\frac{p_{j}}{w_{j}} .
$$

Sort the items in decreasing order of efficiency, then go through the items in this order adding them one-by-one provided that the capacity constraint is not violated thereby. "

Algorithm 2 (Minimization Knapsack problem): "For every item $j \in \mathcal{N}$, denote with $e_{j}$ the weight to profit ratio, i.e.,

$$
e_{j}:=\frac{w_{j}}{p_{j}} .
$$

Sort the items in decreasing order of efficiency, then go through the items in this order adding them one-by-one, unless $\sum_{j=1}^{L} w_{j} x_{j} \geq C_{\min }$ is satisfied."

\section{A. Special Knapsack Features of DTAS}

In DTAS systems, the set $\mathcal{N}$ can be considered as the set of all available system branches, excluding $b_{0}$. This branch-set is denoted by $\mathcal{R}$, i.e., $\mathcal{R}=\left\{b_{1}, \ldots, b_{L}\right\}$. Considering that, in general, the coefficients $p_{j}$ and $w_{j}$ correspond to a performance and energy consumption metric respectively, the following are 
the main points in which DTAS systems differ from the typical knapsack applications:

- In DTAS systems, the total weight capacity does not represent a strictly fixed value with a physical sense, as it occurs in the majority of knapsack applications. On the contrary, it reflects the concept of limiting the total number of relaying terminals and the extra energy consumed to only a single user's avail.

- The amount of time needed for the algorithm computation in DTAS systems is very important, since it has to be small enough in order not to cause any significant delay in packet transmission.

- In DTAS systems, the direct branch $b_{0}$ is always activated since this does not entail any extra consumed energy.

Consequently, Algorithm 1 and Algorithm 2 (slightly modified in order to always include $b_{0}$ ) yield a well-balanced tradeoff between contribution to the total performance and total energy consumption, provided that the energy consumed by the system as a whole does not exceed a predefined threshold.

\section{DTAS IMPLEMENTATION}

\section{A. Average Relay Power Consumption}

The energy $\mathcal{E}_{j}$ that the relay $R_{j}$ consumes per unit time consists in general of two parts: The power consumed by the transmitter/receiver circuitry, including the power needed for signal reception, and the power consumed for amplification. Naturally, the former part is very small compared to the latter, and it is thus neglected. In the time-orthogonal AF scenario described in Section II, the relay passes the received signal through an analog delay line and retransmits it at another timeslot. Therefore, $\mathcal{E}_{j}$ can be defined as

- the difference in the instantaneous transmitting and received power when this difference is positive (or equivalently, when $G_{j}>1$ )

- zero, otherwise. This stems from the fact that, when $G_{j} \leq 1$, the resultant signal attenuation can be achieved by utilizing a passive electronic circuit (e.g., a voltage divider).

Using the notation $(\cdot)^{+}=\max (\cdot, 0)$, we write $\mathcal{E}_{j}$ as $\mathcal{E}_{j}=$ $\left(P_{j, \text { out }}-P_{j, \text { in }}\right)^{+}$, where $P_{j, \text { in }}$ is the signal power at the input of $R_{j}$, for which it holds $P_{j, i n}=P_{j, \text { out }} / G_{j}^{2}$. Therefore, $\mathcal{E}_{j}$ can be written as

$$
\mathcal{E}_{j}=P_{j, \text { out }}\left(1-P_{S} a_{S j}^{2} / P_{j, \text { out }}-N_{0} / P_{j, \text { out }}\right)^{+} .
$$

Averaging over the Nakagami-m distribution [17, eq. (2.21)], the average energy consumed by $R_{j}$ can be approximated in the medium and high SNR regime (where we can ignore the last term in (8)) as

$$
\begin{aligned}
& E\left[\mathcal{E}_{j}\right] \approx \int_{0}^{\frac{P_{j, \text { out }}}{P_{S}}} \frac{\left(P_{j, \text { out }}-P_{S} x\right) m_{S j}^{m_{S j}} x^{m_{S j}-1} e^{-\frac{m_{S j} x}{\Omega_{S j}}}}{\Omega_{S j}^{m_{S j}} \Gamma\left(m_{S j}\right)} d x \\
&= P_{j, \text { out }}-P_{S} \Omega_{S j}+\frac{m_{S j}+1}{\Gamma\left(m_{S j}+2\right)} \\
& \times\left[P_{S} \Omega_{S j} \Gamma\left(m_{S j}+1, \frac{m_{S j} P_{j, \text { out }}}{P_{S} \Omega_{S j}}\right)\right. \\
&\left.\quad-m_{S j} P_{j, \text { out }} \Gamma\left(m_{S j}, \frac{m_{S j} P_{j, \text { out }}}{P_{S} \Omega_{S j}}\right)\right]
\end{aligned}
$$

where $E[\cdot]$ denotes expectation, $m_{S j}$ represents the Nakagami- $m$ parameter of the $S-R_{j} \operatorname{link}, \Gamma(\cdot)$ and $\Gamma(\cdot, \cdot)$ stand for the gamma and incomplete gamma functions defined in [18, eq. (8.310.1)] and [18, eq. (8.350.2)], respectively, and $\Omega_{S j}=E\left[a_{S j}^{2}\right]$.

\section{B. Average Bit Error Probability (ABEP) Minimization Under Energy Consumption Constraints}

Let us denote with $\mathcal{R}_{i}, i=1, \ldots, 2^{L}$ the $i_{\text {th }}$ subset of $\mathcal{R}$; moreover, let $\mathcal{U}_{i}$ represent the union of $\mathcal{R}_{i}$ and $\left\{b_{0}\right\}$, i.e., $\mathcal{U}_{i}=\mathcal{R}_{i} \cup\left\{b_{0}\right\}$, and let $\mathcal{S}$ be the set consisting of all $\mathcal{U}_{i}$, i.e., $\mathcal{S}=\left\{\left\{b_{0}\right\} \cup \mathcal{R}_{i}: \mathcal{R}_{i} \in \mathcal{P}(\mathcal{R})\right\}$, where $\mathcal{P}(\cdot)$ stands for the power set of its argument. Moreover, let $X_{\mathcal{U}_{i}}$ represent the SNR at the MRC output when the branches of $\mathcal{U}_{i}$ are activated, i.e., $X_{\mathcal{U}_{i}}=\sum_{m: b_{m} \in \mathcal{U}_{i}} \gamma_{m}$. Denote by $f_{\gamma_{j}}(\cdot)$, $j=0,1, \ldots, L$, and $f_{X_{\mathcal{U}_{i}}}(\cdot), i=1, \ldots, 2^{L}$, the PDF of $\gamma_{j}$ and $X_{\mathcal{U}_{i}}$ respectively. Since the total energy consumption is constrained, the weight $w_{j}$ of the $b_{j}$ branch is the (long-term) energy consumed by the corresponding relay per unit time, i.e.,

$$
w_{j}=E\left[\mathcal{E}_{j}\right]
$$

The conditional bit error probability (BEP), conditioned on the SNR $\gamma$, assuming DBPSK modulation, is given by

$$
P_{r}(E \mid \gamma)=A \exp (-B \gamma)
$$

where $A, B$ equal to $1 / 2$ and 1 respectively. Likewise, (11) represents an approximation of the BEP of the $M$-PSK and $M$-QAM signal modulations on an AWGN channel; in such case, $A$ and $B$ are derived by fitting the exact conditional BEP curve to the approximated BEP of (11) (see e.g., [19]). For instance, for the BPSK case we found via numerical evaluations that $A$ and $B$ are approximately equal to 0.2568 and 1.2 respectively, when $\gamma$ lies in the interval $[0 \mathrm{~dB}, 20 \mathrm{~dB}]$.

Lemma 1: The coefficients (profits) in the traditional knapsack problem (eq. (4)) that minimize the ABEP for the DBPSK, $M$-PSK and $M$-QAM signal modulations are

$$
p_{j}=\log _{\beta}\left[\frac{1}{\mathcal{M}_{\gamma_{j}}(-B)}\right], j=1, \ldots, L,
$$

where $\beta>1$ and $\mathcal{M}_{\gamma_{j}}(s)$ is the moment generating function (MGF) of $\gamma_{j}$ defined as

$$
\mathcal{M}_{\gamma_{j}}(s) \triangleq E\left[\exp \left(s \gamma_{j}\right)\right]=\int_{0}^{\infty} \exp \left(s \gamma_{j}\right) f_{\gamma_{j}}\left(\gamma_{j}\right) d \gamma_{j}
$$

Proof: Suppose that the set $\mathcal{U}_{\kappa}$ is selected among all $\mathcal{U}_{i} \in \mathcal{S}$, by substituting (10) and (12) into (4). It holds that

$$
\begin{aligned}
& \mathcal{U}_{\kappa}=\arg \max _{\sum_{m: b_{m} \in \mathcal{U}_{i}} w_{m} \leq C_{\max }} \sum_{m: b_{m} \in \mathcal{U}_{i}} \log _{\beta}\left[\frac{1}{\mathcal{M}_{\gamma_{m}}(-B)}\right] \\
& =\arg \max _{\sum_{m: b_{m} \in \mathcal{U}_{i}} w_{m} \leq C_{\max }} \log _{\beta}\left[\prod_{m: b_{m} \in \mathcal{U}_{i}} \frac{1}{\mathcal{M}_{\gamma_{m}}(-B)}\right] \\
& =\arg \max _{\sum_{m: b_{m} \in \mathcal{U}_{i}} w_{m} \leq C_{\max }} \prod_{m: b_{m} \in \mathcal{U}_{i}} \frac{1}{\mathcal{M}_{\gamma_{m}}(-B)}
\end{aligned}
$$


TABLE I

DTAS Traditional KNAPSACK Problem: ABEP AND NORMALIZEd TOTAL POWER CONSUMPTION

\begin{tabular}{||c|c|c|c|c|c|}
\hline \multicolumn{1}{|c|}{ ABEP / Consumption } \\
\hline \multirow{3}{*}{$C_{\text {max }}=1.6$} & $\begin{array}{c}\text { Available } \\
\text { Relays }\end{array}$ & Proposed Scheme & 1 Relay: Lowest ABEP & 1 Relay: Highest Eff. & \multirow{2}{*}{ All Relays } \\
\cline { 2 - 7 } & 5 & $1.29 \times 10^{-3} / 1.515$ & $8.44 \times 10^{-2} / 0.258$ & $8.44 \times 10^{-2} / 0.258$ & $5.16 \times 10^{-4} / 1.958$ \\
\cline { 2 - 7 } & 10 & $6.23 \times 10^{-4} / 1.599$ & $8.44 \times 10^{-2} / 0.258$ & $8.44 \times 10^{-2} / 0.258$ & $1.61 \times 10^{-5} / 3.907$ \\
\cline { 2 - 7 } & 15 & $4.07 \times 10^{-4} / 1.393$ & $7.53 \times 10^{-2} / 0.196$ & $7.53 \times 10^{-2} / 0.196$ & $1.46 \times 10^{-7} / 6.193$ \\
\cline { 2 - 7 } & 20 & $2.80 \times 10^{-4} / 1.560$ & $7.53 \times 10^{-2} / 0.196$ & $7.53 \times 10^{-2} / 0.196$ & $2.22 \times 10^{-9} / 7.900$ \\
\hline \hline \multirow{3}{*}{$C_{\max }=6$} & 5 & $5.16 \times 10^{-4} / 1.958$ & $8.44 \times 10^{-2} / 0.258$ & $8.44 \times 10^{-2} / 0.258$ & $5.16 \times 10^{-4} / 1.958$ \\
\cline { 2 - 7 } & 10 & $1.61 \times 10^{-5} / 3.907$ & $8.44 \times 10^{-2} / 0.258$ & $8.44 \times 10^{-2} / 0.258$ & $1.61 \times 10^{-5} / 3.907$ \\
\cline { 2 - 7 } & 15 & $3.73 \times 10^{-7} / 5.359$ & $7.53 \times 10^{-2} / 0.196$ & $7.53 \times 10^{-2} / 0.196$ & $1.46 \times 10^{-7} / 6.193$ \\
\cline { 2 - 7 } & 20 & $3.39 \times 10^{-8} / 5.467$ & $7.53 \times 10^{-2} / 0.196$ & $7.53 \times 10^{-2} / 0.196$ & $2.22 \times 10^{-9} / 7.900$ \\
\hline
\end{tabular}

The last equation follows from its preceding since $\log _{\beta}(x)$ is an increasing function of $x$, for $x>0$ and for any $\beta>1$. Using (13), (14) can be rewritten as

$$
\begin{gathered}
\mathcal{U}_{\kappa}=\arg \max _{\sum_{m: b_{m} \in \mathcal{U}_{i}} w_{m} \leq C_{\max }} \prod_{m: b_{m} \in \mathcal{U}_{i}} \frac{1}{\int_{0}^{\infty} e^{-B \gamma_{m}} f_{\gamma_{m}}\left(\gamma_{m}\right) d \gamma_{m}} \\
=\arg \min _{\sum_{m: b_{m} \in \mathcal{U}_{i}} w_{m} \leq C_{\max }}\left[\int_{0}^{\infty} \int_{0}^{\infty} \cdots \int_{0}^{\infty} e^{-B \sum_{m: b_{m} \in \mathcal{U}_{i}} \gamma_{i}}\right. \\
\left.=\arg \prod_{\sum_{\sum_{\mathcal{U}_{i} \in \mathcal{S}} w_{m} \in \mathcal{U}_{i}} w_{m} \leq C_{\max }} \int_{0}^{\infty}\left[f_{\gamma_{m}}\left(\gamma_{i}\right) d \gamma_{m}\right]\right] \\
A e^{-B X_{\mathcal{U}_{i}}} f_{X_{\mathcal{U}_{i}}}\left(X_{\mathcal{U}_{i}}\right) d X_{\mathcal{U}_{i}} .
\end{gathered}
$$

Since (11) implies that the integral in (15) represents the exact or the approximated ABEP for the DBPSK, $M$-PSK and $M$ QAM signal modulations, the proof has been completed.

Therefore, the problem of optimizing the ABEP under total energy consumption constraints reduces to substituting (10) and (12) into (4).

\section{Total Energy Consumption Minimization Under ABEP Constraints}

In such case, the coefficients $p_{j}$, in (5) represent the energy consumed by the relay corresponding to the branch $b_{j}$, i.e.,

$$
p_{j}=E\left[\mathcal{E}_{j}\right] \text {, }
$$

where $E\left[\mathcal{E}_{j}\right]$ is given in (9).

Lemma 2: By setting the coefficients $w_{j}$ in the minimization knapsack problem (eq. (5)) as

$$
w_{j}=\log _{\beta}\left[\frac{1}{\mathcal{M}_{\gamma_{j}}(-B)}\right], j=0,1, \ldots, L,
$$

where $\beta>1$, we ensure that the ABEP for the DBPSK, $M$-PSK and $M$-QAM signal modulations does not exceed a predefined threshold $\delta$ (if possible ${ }^{2}$ ).

\footnotetext{
${ }^{2}$ It is evident that Lemma 2 does not hold when the activation of all the available relays leads to an ABEP which is greater than the predefined threshold $\delta$.
}

Proof: Assume that the set $\mathcal{U}_{\kappa}$ is selected among all $\mathcal{U}_{i} \in \mathcal{S}$, by substituting (16) and (17) into (5). Then,

$$
\begin{aligned}
\sum_{j: b_{j} \in \mathcal{U}_{\kappa}} w_{j} & =\sum_{j: b_{j} \in \mathcal{U}_{\kappa}} \log _{\beta}\left[\frac{1}{\mathcal{M}_{\gamma_{j}}(-B)}\right] \\
& =\log _{\beta}\left[\prod_{j: b_{j} \in \mathcal{U}_{\kappa}} \frac{1}{\mathcal{M}_{\gamma_{j}}(-B)}\right] .
\end{aligned}
$$

Substituting (13) into (18) yields

$$
\begin{aligned}
& \sum_{j: b_{j} \in \mathcal{U}_{\kappa}} w_{j} \\
&=\log _{\beta}\left[\frac{1}{\left.\int_{0}^{\infty} \cdots \int_{0}^{\infty} e^{-B \sum_{j: b_{j} \in \mathcal{U}_{\kappa} \gamma_{j}} \prod_{j: b_{j} \in \mathcal{U}_{\kappa}} f_{\gamma_{j}}\left(\gamma_{j}\right) d \gamma_{j}}\right]}\right. \\
&=\log _{\beta}\left[\left(\int_{0}^{\infty} \exp \left(-B X_{\mathcal{U}_{\kappa}}\right) f_{X_{\mathcal{U}_{\kappa}}}\left(X_{\mathcal{U}_{\kappa}}\right) d X_{\mathcal{U}_{\kappa}}\right)^{-1}\right]
\end{aligned}
$$

Since (5) ensures that $\sum_{j: b_{j} \in \mathcal{U}_{\kappa}} w_{j} \geq C_{\min }$, from (19) we obtain

$$
A \int_{0}^{\infty} \exp \left(-B X_{\mathcal{U}_{k}}\right) f_{X_{\mathcal{U}_{k}}}\left(X_{\mathcal{U}_{k}}\right) d X_{\mathcal{U}_{k}} \leq A \beta^{-C_{\min }}
$$

Thus, we realize that the system's ABEP for the DBPSK, $M$ PSK and $M$-QAM signal modulations can be upper-bounded by a predefined value $\delta=A \beta^{-C_{\text {min }}}$.

Consequently, the problem of minimizing the total energy consumption in an average sense, provided that the ABEP is below a given threshold reduces to substituting (16) and (17) into (5). From the above, it is easy to understand that if, for example, the ABEP needs to be kept below the value of $\delta=10^{-z}$ in a BPSK application, then the value $C_{\min }$ in (5) is substituted with $C_{\min } \approx \log _{\beta}[A / \delta] \approx \log _{\beta}\left[0.2568 \cdot 10^{z}\right]$.

\section{NUMERICAL EXAMPLES AND DISCUSSION}

In order to illustrate the performance of the proposed scheme, an extensive set of numerical examples is performed, using the MGF-based approach for the ABEP given in [17, eq. (5.3)]. BPSK modulation is assumed, and the fading on the $S-D$ and on each $S-R_{j}$ and $R_{j}-D$ channel is considered 
TABLE II

DTAS Minimization KNAPSACK PRoblem: ABEP AND NORMALIZEd TOTAL POWER CONSUMPTION

\begin{tabular}{|c|c|c|c|c|c|}
\hline \multicolumn{9}{|c|}{ ABEP / Consumption } \\
\hline \multirow{3}{*}{$\delta=10^{-4}$} & $\begin{array}{c}\text { Available } \\
\text { Relays }\end{array}$ & Proposed Scheme & 1 Relay: Lowest ABEP & 1 Relay: Highest Eff. & All Relays \\
\cline { 2 - 7 } & 5 & $5.16 \times 10^{-4} / 1.958$ & $8.44 \times 10^{-2} / 0.258$ & $9.71 \times 10^{-2} / 0.294$ & $5.16 \times 10^{-4} / 1.958$ \\
\cline { 2 - 7 } & 10 & $8.34 \times 10^{-5} / 3.112$ & $8.44 \times 10^{-2} / 0.258$ & $9.71 \times 10^{-2} / 0.294$ & $1.61 \times 10^{-5} / 3.907$ \\
\cline { 2 - 7 } & 15 & $1.73 \times 10^{-5} / 2.944$ & $7.53 \times 10^{-2} / 0.196$ & $8.61 \times 10^{-2} / 0.214$ & $1.46 \times 10^{-7} / 6.193$ \\
\cline { 2 - 7 } & 20 & $2.91 \times 10^{-5} / 2.250$ & $7.53 \times 10^{-2} / 0.196$ & $8.61 \times 10^{-2} / 0.214$ & $2.22 \times 10^{-9} / 7.900$ \\
\hline \hline \multirow{3}{*}{$\delta=10^{-6}$} & 5 & $5.16 \times 10^{-4} / 1.958$ & $8.44 \times 10^{-2} / 0.258$ & $9.71 \times 10^{-2} / 0.294$ & $5.16 \times 10^{-4} / 1.958$ \\
\cline { 2 - 7 } & 10 & $1.61 \times 10^{-5} / 3.907$ & $8.44 \times 10^{-2} / 0.258$ & $9.71 \times 10^{-2} / 0.294$ & $1.61 \times 10^{-5} / 3.907$ \\
\cline { 2 - 7 } & 15 & $1.71 \times 10^{-7} / 4.675$ & $7.53 \times 10^{-2} / 0.196$ & $8.61 \times 10^{-2} / 0.214$ & $1.46 \times 10^{-7} / 6.193$ \\
\cline { 2 - 7 } & 20 & $1.58 \times 10^{-7} / 3.971$ & $7.53 \times 10^{-2} / 0.196$ & $8.61 \times 10^{-2} / 0.214$ & $2.22 \times 10^{-9} / 7.900$ \\
\hline
\end{tabular}

independent and Nakagami- $m$ distributed, with the fading parameter $m$ being a random variable (RV) uniformly distributed in the interval $[1,2.5]$. The average values of the fading attenuation on the direct $S-D$, and each $S-R_{j}$ and $R_{j}-D$ channel $\left(\Omega_{S D}, \Omega_{S j}\right.$ and $\Omega_{D j}$ respectively) are considered continuous independent and identically distributed (i.i.d.) lognormal RVs, with mean and standard deviation 0.25 and 0.1 respectively for the $S-D$, and 0.5 and 0.2 respectively for the $S-R_{j}$ and $R_{j}$ $D$ channels. Moreover, in our examples we assume that the relays' transmission powers are identical with the source's, and that these transmission powers are normalized to unity i.e., $P_{j \text {,out }}=P_{S}=1$ for all $j \in\{1, \ldots, L\}^{3}$; for this reason, in the sequel the term normalized will denote normalization with respect to $P_{S}$. The noise power $N_{0}$ was set equal to ten percent of $P_{S}$, i.e. $N_{0}=0.1$.

The main advantage of the traditional and the minimization knapsack problem utilization in DTAS systems is presented in Tables I and II, respectively. In these Tables, the proposed model is compared, in terms of ABEP and normalized total power consumption, with three different schemes: a) the "all participate" one, involving activation of all the available branches, b) the scheme where a single branch $b_{\eta}$, the one with the highest efficiency is activated through $\eta=\arg \max _{j=1, \ldots, L} e_{j}$, where $e_{j}$ defined in (6) and (7) for the traditional and minimization knapsack formulation, respectively, and c) the scheme where only the branch entailing the lowest long-term ABEP is activated. In the latter model, Lemma 1 implies that the branch $b_{\kappa}$ is activated if $\kappa=\arg \min _{j=1, \ldots, L} \mathcal{M}_{\gamma_{j}}(-B)$, where $B \approx 1.2$ for the BPSK case.

In general, the proposed model appears to achieve a wellbalanced tradeoff between error performance and energy consumption, which is also controllable in the sense that the proposed scheme performs in a way similar to the case where a single or all the available relays are activated. This is determined by the energy consumption constraint $C_{\max }$, or by the equivalent $\mathrm{ABEP}$ one $\delta$, corresponding to the traditional and the minimization knapsack problem, respectively. If, for example, the value of $C_{\max }$ is small, or the value of $\delta$ is high enough so that only one relay is activated, the knapsack

\footnotetext{
${ }^{3}$ It is evident that the same problem can be also applied in the case where the relays' transmission powers are determined by a power allocation procedure and are not necessarily identical with one another.
}

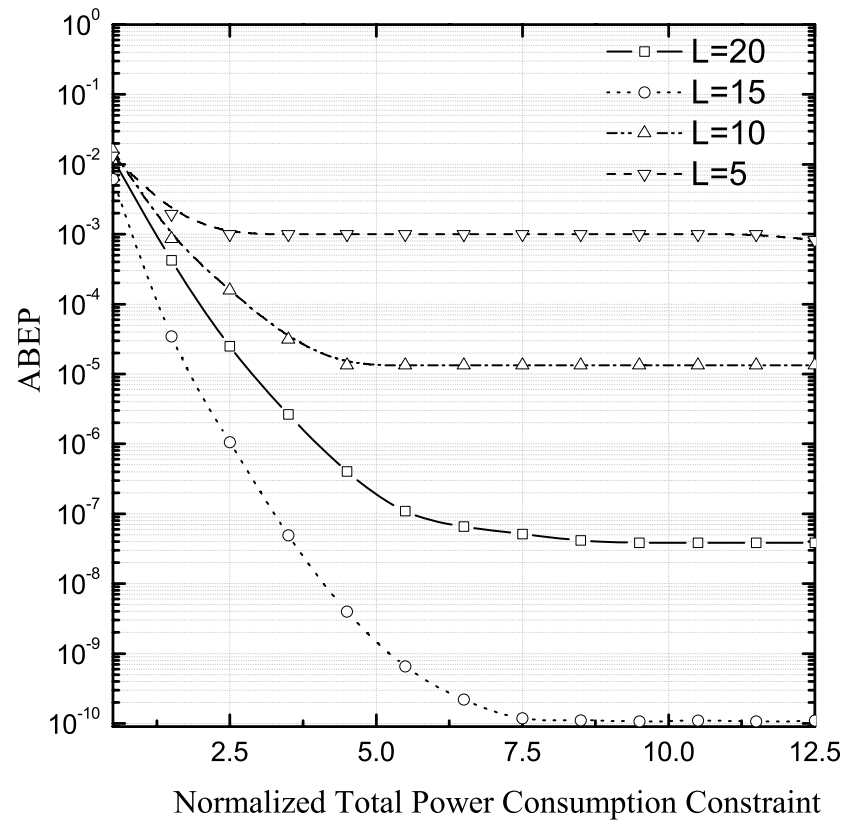

Fig. 1. Traditional knapsack problem: ABEP vs energy consumption constraint.

model reduces to the scheme where the only cooperating node is that with the highest efficiency. Likewise, the knapsack scheme can act as an "all participate" selection model, by setting high or small values for $C_{\max }$ or $\delta$, respectively. Thus, it is evident that the proposed selection method allows the system administrator to easily adapt the system's performanceconsumption tradeoff, according to its needs.

Fig. 1 depicts the system's ABEP versus the normalized value of $C_{\max }$, for some $L$ assumptions, when the traditional knapsack problem (eq. (4)) is utilized. As expected, relaxing the power consumption constraint results in generally better ABEP performance; however, we notice that a floor point on the ABEP exists, which corresponds to the case where all the available relays are activated. That is, increasing $C_{\max }$ more than a certain point does not lead to lower ABEP since the number of active relays remains the same.

Regarding the minimization knapsack problem (eq. (5)), similar observations about the effect of the performance constraint on the total consumed power can be extracted from 


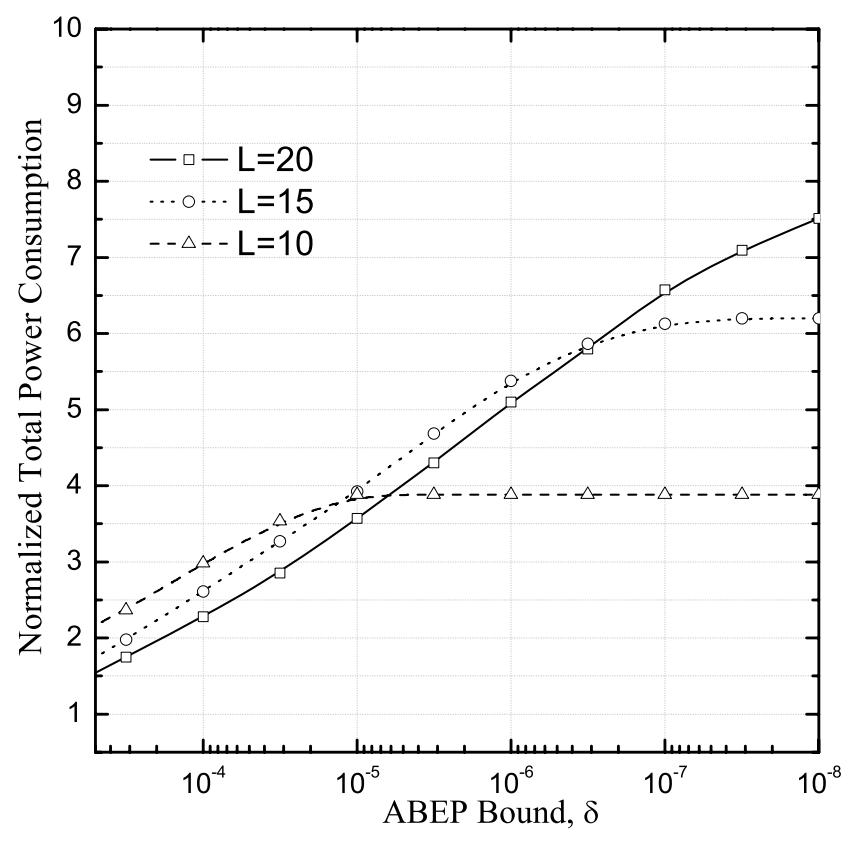

Fig. 2. Minimization knapsack problem: energy consumption vs ABEP bound.

Fig. 2. Specifically, we notice that increasing $\delta$ results in an increase in the total consumed power. Additionally, a ceiling point on the total consumed power exists, which is reached for as higher values of $\delta$ as larger the number of available relays. This is the reason why a crossing point between any pair of curves in Fig. 2 is observed, since after a certain value of $\delta$ the systems with relatively higher values of $L$ can reach the target ABEP, whereas the ones with lower $L$ cannot.

\section{ACKNOWLEDGEMENTS}

The authors would like to thank Mr. Lazaros Sidiropoulos for helpful discussions, and the anonymous reviewers whose constructive comments have contributed to improve the quality of this letter.

\section{REFERENCES}

[1] S. Kaviani and C. Tellambura, "Closed-form BER analysis for antenna selection using orthogonal space-time block codes," IEEE Commun. Lett., vol. 10, pp. 704-706, Oct. 2006.
[2] D. A. Gore and A. J. Paulraj, "MIMO antenna subset selection with space-time coding," IEEE Trans. Signal Processing, vol. 50, pp. 25802588, Oct. 2002.

[3] X. Cai and G. Giannakis, "Performance analysis of combined transmit selection diversity and receive generalized selection combining in Rayleigh fading channels," IEEE Trans. Wireless Commun., vol. 3, pp. 1980-1983, Nov. 2004.

[4] J. N. Laneman, D. N. C. Tse, and G. W. Wornell, "Cooperative diversity in wireless networks: Efficient protocols and outage behavior," IEEE Trans. Inform. Theory, vol. 50, pp. 3062-3080, Dec. 2004.

[5] A. Sendonaris, E. Erkip, and B. Aazhang, "User cooperation diversityPart I: System description,” IEEE Trans. Commun., vol. 51, pp. 19271938, Nov. 2003.

[6] M. O. Hasna and M. S. Alouini, "End-to-end performance of transmission systems with relays over Rayleigh fading channels," IEEE Trans. Wireless Commun., vol. 2, pp. 1126-1131, Nov. 2003.

[7] P. A. Anghel and M. Kaveh, "Exact symbol error probability of a cooperative network in a Rayleigh-fading environment," IEEE Trans. Wireless Commun., vol. 3, pp. 1416-1421, Sept. 2004.

[8] A. Ribeiro, X. Cai, and G. Giannakis, "Symbol error probabilities for general cooperative links," IEEE Trans. Wireless Commun., vol. 4, pp. 1264-1273, May 2005.

[9] J. Luo, R. S. Blum, L. J. Greenstein, and A. M. Haimovich, "Linkfailure probabilities for practical cooperative relay networks," in Proc. IEEE vehicular Technology Conference (VTC'05-Spring), Stockholm, Sweden, May 2005.

[10] A. Bletsas, A. Khisti, D. P. Reed, and A. Lippman, "A simple cooperative diversity method based on network path selection," IEEE J. Select. Areas Commun., vol. 24, pp. 659-672, Mar. 2006.

[11] E. Beres and R. Adve, "On selection cooperation in distributed networks," in Proc. Conference on Information Sciences and Systems (CISS 06), Princeton, NJ, Mar. 2006.

[12] Y. Zhao, R. Adve, and T. J. Lim, "Symbol error rate of selection amplifyand-forward relay systems," IEEE Commun. Lett., vol. 10, pp. 757-759, Nov. 2006.

[13] R. Madan, N. B. Mehta, A. F. Molisch, and J. Zhang, "Energy-efficient cooperative relaying over fading channels with simple relay selection," in Proc. IEEE Global Communications Conference (GLOBECOM'06), San Francisco, CA, Nov. 2006.

[14] S. Martello and P. Toth, Knapsack Problems-Algorithms and Computer Implementations. New York: Wiley, 1990.

[15] J. Boyer, D. D. Falconer, and H. Yanikomeroglu, "Multihop diversity in wireless relaying channels," IEEE Trans. Commun., vol. 52, pp. 18201830, Oct. 2004.

[16] R. You, H. Li, and Y. Bar-Ness, "Diversity combining with imperfect channel estimation," IEEE Trans. Commun., vol. 53, pp. 1655-1662, Oct. 2005.

[17] M. K. Simon and M.-S. Alouini, Digital Communication over Fading Channels, 2nd ed. New York: Wiley, 2005.

[18] I. S. Gradshteyn and I. M. Ryzhik, Table of Integrals, Series, and Products, 6th ed. New York: Academic, 2000.

[19] Y.-Ko and M.-S. Alouini, "Estimation of Nakagami- $m$ fading channel parameters with application to optimized transmitter diversity systems," IEEE Trans. Wireless Commun., vol. 2, pp. 250-259, Mar. 2003. 